\title{
Historical Note
}

\section{The Trend of Research on Number Theory in Bengal and Bihar during the $20^{\text {th }}$ Century}

\author{
Purabi Mukherji*
}

(Received 27 August 2018; revised 22 January 2019)

\begin{abstract}
The research on number theory in India during the modern times started with the advent of Srinivasa Ramanujan. His theories, conjectures and questions on various topics of number theory inspired mathematicians both at home and abroad. During the twentieth Century, inspired and influenced by Ramanujan notable research was done in South India and Punjab. In the Eastern part of the country, some work was done by a handful of number theorists. They are not so well-known. In this article, an attempt has been made to bring to the fore the research work done in Bengal and Bihar during the $20^{\text {th }}$ Century. The mathematicians of these two states worked mainly on problems which were first initiated by Ramanujan. Most of the published works were on Ramanujan's function $\tau(\mathrm{n})$, theory of partitions and continued fractions. From the historical perspective there is a necessity to highlight these contributions.
\end{abstract}

Key words: Congruence, Continued fractions, Theory of partitions, Ramanujan's function, Waring's problem.

\section{InTRODUCTION}

The great German mathematician Felix Klein had said: "Plato said 'God is a geometer'. Jacobi changed this to 'God is an arithmetician'. Then came Kronecker and fashioned the memorable expression 'God created the natural numbers, and all the rest is the work of man." Number Theory or theory of numbers has always held a unique position in the world of mathematics. Because of its fundamental place in the discipline, number theory is sometimes referred to as 'The Queen of Mathematics'.

\subsection{Historical Prelude}

Since ancient times nearly every century has been a witness to new and fascinating discoveries related to the properties of numbers. India had a long tradition of research on number theory since the ancient times. Famous and eminent Indian mathematicians like Brahmagupta, Bhaskaracharya, Jayadeva and others made notable contributions in this area. In the Western world too all-time great mathematicians like Euler, Gauss, Lagrange, Jacobi and many others have contributed to this area.

\subsection{Definition}

Before proceeding any further, the natural question would be 'what is number theory?' The answer is that number theory is a branch of mathematics which deals with the properties and relationships of numbers, especially the positive integers. The two major classifications of number theory are analytic number theory and algebraic

*CA 17, Sector I, Salt Lake, Kolkata-700064, Email: rubym1945@gmail.com. 
number theory. Analytic number theory uses methods from mathematical analysis to solve problems about integers. On the other hand algebraic number theory is a major branch of the discipline that studies algebraic structures related to algebraic integers.

\section{Indian Scenario in the $20^{\text {Th }}$ Century}

In India, in the twentieth Century, the advent of Srinivasa Ramanujan (1887-1920) led to pathbreaking researches in the theory of numbers. Ramanujan himself made substantial contributions on analytic number theory. His theories, his conjectures, the questions that he raised made world-wide impact and generations of international and Indian mathematicians were inspired and influenced by him. The South Indian school of number theory produced highly acclaimed number theorists like S S Pillai, T Vijayaraghavan. The Punjab school produced first grade researchers like S Chowla, Hansraj Gupta and R P Bambah. The states of Bengal and Bihar in contrast did not produce any such superlative scholars. But these two states did produce a handful of sincere mathematicians who did research in area of theory of numbers to the best of their ability. From the historical perspective it would be fair to highlight their contributions. It is to be noted that the earliest known research publication in this area from Bengal was by $\mathrm{B} \mathrm{N}$ Seal (Brojendra Nath Seal) in 1891. He had written "A Memoir on the Coefficient of Number". But since that was published in the nineteenth Century, it is not within the purview of this article.

\section{Trend of Research in Bengal}

In the $20^{\text {th }}$ Century, the earliest known research publication from Bengal was once again by $\mathrm{B} \mathrm{N}$ Seal. B N Seal (1864-1938) was a humanist philosopher and adorned the prestigious chair of the King George V Professor of Philosophy at the University of Calcutta. But he had an inherent fascination for number theory. His paper entitled
'The equation of digits; being an elementary application of a principle of numerical grouping to the solution of numerical equation ' $(B C M S, 10$, 1919, pp. 99-123) was the first published paper on theory of numbers from the state of Bengal. Around the same time H Datta published a paper titled 'On some properties of natural numbers' (BCMS, 10, 1919, pp. 229-238). But after 1919, almost for more than a decade no notable publication has been noticed. In 1929, S C Mitra incorporated a particular result on number theory (which was established by him) in his Ph.D. thesis. This result was published three years later as a paper entitled 'On the proof of a result given by Ramanujan about the complex multiplication of elliptic function' (BCMS, 24, 1932, pp. 135-136). In this paper, S C Mitra has given the proof of a result about complex multiplication of elliptic functions. Ramanujan had stated this result without a proof, in his paper titled 'Modular equations and applications to $\pi$ ' (QJM, 45, 1914, pp. 350-372). It is noteworthy that the first investigation on a topic related to Ramanujan's work was carried out in Bengal nine years after the demise of the iconic mathematician. Then a few notable incidents took place, which shaped and helped the development of research on number theory in Bengal.

Firstly the famous mathematician and number theorist from South India, T Vijayaraghavan (1902-1955) left the Aligarh Muslim University and joined the Dacca University in erstwhile East Bengal (present day Bangladesh) in 1931. Vijayaraghavan was mentored by K Ananda Rau at Madras University, trained at Oxford University by G H Hardy and had close interaction with world famous mathematician A Weil during his brief stint at the Aligarh Muslim University. He joined the Dacca University as a Lecturer in the Department of Mathematics and worked there till 1946.

It was during this period that D P Banerjee, a Lecturer of Mathematics in a small College named A M College at Mymensingh in East Bengal got 
inspired by Vijayaraghavan and started doing serious research on various topics connected to analytic number theory. D P Banerjee published his first paper titled 'On the solution of the 'easier' Waring problem' (BCMS, 34, 1942, pp. 197-199) (Appendix 1). Subsequently in a series of two papers Banerjee in 1942, investigated the congruence properties of Ramanujan's function $\tau$ (n) (Appendix 2). The first of these papers was published in $J L M S(17,1942$, pp. 144-145) and the second one in PNAS (India) (Sect. A, 12, 1942, pp. 149-150). In the above two papers, the author has stated that Ramanujan's function $\tau$ (n) satisfies many congruence properties and many of them have been proved by Ramanujan himself as well as by Mordell, Watson and others. But a few new congruence properties which perhaps had not been noticed earlier have been solved by him. The next year in 1943, Banerjee wrote a paper on rational solutions of a Diophantine Equation (Appendix 3 ) of a special type. The paper was published in 1943 (PBMS, N.S, 5, 1943, pp. 29-30). In 1944, D P Banerjee published two papers titled 'On some formulae in analytic theory of numbers' ( $B C M S$, 36,1944 , pp. 49-50) and 'On some formulae in analytic theory of numbers II' (BCMS, 36, 1944, pp. 107-108).

In these two papers, in continuation to certain formulas of analytic number theory which were considered by Ramanujan, Estermann and Hardy as stated in the collected papers of Ramanujan (Ed. by G H Hardy, P V Seshu Aiyer and B M Wilson, Cambridge University Press, Cambridge, 1927), Banerjee added a few more interesting formulas to the list. In his paper titled 'On the application of congruence properties of Ramanujan's function to certain quarternary form' $(B C M S, 37,1945, \mathrm{pp}$. 24-26), D P Banerjee claims to have proved certain congruence properties of Ramanujan's function $\tau(\mathrm{n})$. It may be noted that in this paper, the author also considered new and interesting applications of the congruence properties to the possibility of finding applications to certain quarternary (having four parts) equations. The same year Banerjee published a paper entitled 'On a theorem in the theory of partitions' (BCMS, 37, 1945, pp. 113-114). Years later in 1964 he published another paper related to some identities in the theory of partitions (Appendix 4) (PNAS, India, Sect. A, 34, 1964, pp. 68-73). In his paper titled 'On the divisors of numbers' (BCMS, 39, 1947, pp. 57-58), it may be noted that the excess of the number of divisors of a number $n$ which have the form $\mathrm{m}+1$ over the number of divisors which have the form $4 \mathrm{~m}+3$ had been termed by $\mathrm{J}$ W L Glaisher (1848-1928) as E(n). In the paper mentioned above D P Banerjee considered some new formulas of this and other functions of divisors. In the ten papers of D P Banerjee that have been discussed above, eight of them are connected with Ramanujan's function $\tau(n)$, partition functions and Diophantine equations. These are areas where Srinivasa Ramanujan had pioneered research in India. Banerjee was clearly highly inspired and motivated by the works of the legendary Indian mathematician.

The next important event that enriched research on number theory in Bengal relates to a mathematician named D B Lahiri. D B Lahiri (Deba Brata Lahiri) was born on the $17^{\text {th }}$ February, 1913 in Rangoon, the capital of erstwhile Burma (present day Myanmar). They were originally from East Bengal. But natural disasters, poverty and the onset of the First World War forced the family to migrate to Burma in search of a new homeland. The family of Lahiri was extremely poor and due to precarious financial conditions, the young student had to depend on stipends and scholarships from the tender age of nine to carry on with his school education. He fared well in the School Final Examination in Rangoon but could not qualify for a Government Scholarship, as he was under-aged. Fortunately, an Englishman, L G Owen, who happened to be a Professor of Mathematics, helped him procure adequate stipends. With that financial help D B Lahiri completed his B.Sc. with Honours 
in Mathematics from the Rangoon University in 1933. He stood First among the successful candidates and was awarded a gold medal. With the help of Owen and another Englishman N Lewis, Lahiri became well-trained in the basics of Statistics. In collaboration with Lewis, he coauthored a paper on Demography, which was published in Sānkhya, the Indian Journal of Statistics. P C Mahalanobis, the Editor of the said journal, probably noted the point. After some initial stints at odd jobs, Lahiri was finally recruited at the Indian Statistical Institute (ISI) by Mahalanobis in 1946. In the Indian Statistical Institute he was intensely trained by Mahalanobis, S N Ray and other stalwarts in specialized branches of applied statistics. But famed mathematician R C Bose came to know about Lahiri's passion for number theory. As a school boy in Rangoon, Lahiri had developed a fascination for the theory of numbers. The news of the sudden and untimely death of S Ramanujan had left a lasting impact in Lahiri's mind. Since his young school days, Lahiri was eager and curious to learn about Ramanujan and his contributions in number theory. While at the ISI, Professor R C Bose encouraged Lahiri to carry on his private research work on the theory of numbers and also introduced him to the famous South Indian number theorist S S Pillai. At the initiation and persuasion of F W Levi, S S Pillai had joined the Pure Mathematics Department of the Calcutta University in 1942. D B Lahiri toiled hard on the training on applied statistics that he was receiving at the ISI, but in his spare time, he assiduously carried out his private research on the theory of numbers. As he was always fascinated by Ramanujan and his research on number theory, Lahiri's first paper on theory of numbers was titled 'On Ramanujan's function $\tau(\mathrm{n})$ and divisor function $\sigma_{\mathrm{k}}(\mathrm{n})-\mathrm{I}$ ' $(B C M S, 38$, 1946, pp. 193206). In this particular paper, Lahiri developed a systematic method of studying certain congruence properties of the divisor function $\sigma_{\mathrm{k}}(\mathrm{n})$, the sum of the $k$ th powers of the divisors of $n$. In the same issue of the Bulletin of the Calcutta Mathematical Society, D B Lahiri published another paper titled 'On a type of series involving the partition function with applications to certain congruence relations' (BCMS, 38, 1946, pp. 125-132). He prepared this paper under the supervision of R C Bose. In this paper, Lahiri discovered the recursive congruence properties of the partition function $p(n)$ and showed that the well-known Ramanujan's congruences:

$$
\mathrm{p}(5 \mathrm{~m}+4 \equiv 0(\bmod 5), \mathrm{p}(7 \mathrm{~m}+5) \equiv 0(\bmod 7)
$$
and $\mathrm{P}(11 \mathrm{~m}+6) \equiv 0(\bmod 11)$ can be deduced by the method of induction from the recursive congruences established by him. In 1947, Lahiri published two papers both dealing with Ramanujan's function $\tau$ (n). The first one was an extension of his earlier paper published in 1946 and was titled On Ramanujan's function $\tau(\mathrm{n})$ and divisor function $\sigma_{\mathrm{k}}(\mathrm{n})-$ II' (BCMS, 39, 1947, pp. 33-52). The second paper was written in collaboration with R P Bambah, S Chowla and H Gupta and titled 'Congruence properties of Ramanujan's function $\tau(\mathrm{n})$ '(QJM, Oxford, Ser. 18,1947 , pp. 143-146). It may be noted that in this paper Lahiri had collaborated with the most prominent Indian number theorists of his time. In 1948 and 1949 he published two research papers dealing with non-Ramanujan congruence properties of the partition. After a gap of more than 15 years, Lahiri again started publishing important research papers on various topics of the theory of numbers. Between 1966 and 1971, he published as many as 11 research papers and they were all published in well-known national and international journals. Two of these papers deserve special attention. In the paper titled 'Some congruences of the elementary divisor functions' (AMM, 76 (1-10), 1969, pp. 395-397), Lahiri carried out studies on $\sigma_{\mathrm{k}}(\mathrm{n})$ and also established two interesting theorems involving Euler's function $\varphi(n)$. In the other notable paper titled 'Some restricted partition functions; congruence modulo 3' (PJM, 28.3, 1969, pp. 575-581), the 
author established some congruence relations with respect to modulo 3 for some restricted partition functions. He has also discussed about unrestricted partition functions and stated two theorems involving them. Using the well-known 'pentagonal number theorem' of Euler, he proved the theorems. D B Lahiri published a total 17 research papers in the area of number theory. In the Indian Statistical Institute he was trained in Sample Survey and he was quite famous as a statistician. He was elected a Fellow of the National Academy of Sciences. Research in the theory of numbers was practically a private commitment. But in spite of that, he stands tall as a number theorist of repute in Bengal.

Apart from D B Lahiri, the renowned mathematician Professor R C Bose of ISI contributed a couple of papers on number theory. His paper written in collaboration with S Chowla and C R Rao titled 'On the integral order $(\bmod p)$ of quadratics $x^{2}+a x+b$ with applications to the construction of minimum functions for GF $\left(p^{2}\right)$, and to some number theory result' (BCMS, 36, 1944 , pp. 153-174) is interesting. Here the authors considered polynomials whose coefficients belonged to the ring of integers. In this paper $p$ always denotes an odd prime. Again if $\mathrm{n}$ is the least positive integer such that $x^{n} \equiv$ an integer $(\mathrm{mod}$ $\left.p, x^{2}+a x+b\right)$ then $\mathrm{n}$ is called the 'integral order' of $x^{2}+a x+b(\bmod p)$. In this particular paper, the three authors have deduced a number of interesting theorems about the integral order. R C Bose published another paper titled 'On the construction of affine difference sets', in collaboration with $\mathrm{S}$ Chowla.

The third important event that enriched the number theoretic researches in Bengal relates to S S Pillai of the University of Calcutta. As already mentioned, in 1942. Pillai joined the Pure Mathematics Department of the Calcutta University. Prior to that, S S Pillai and T Vijayaraghavan had a long-standing friendship starting from their student days at Madras
University. When Vijayaraghavan was in Oxford, Professor G H Hardy discussed a problem which arose in the context of a famous paper of $\mathrm{G} \mathrm{H}$ Hardy and S Ramanujan titled 'The normal number of prime factors of a number n' (QJM, Oxford, 48, 1917, pp. 76-92). This problem which Hardy posed to his students at Oxford came to be known as 'Hardy's Problem' and remained unsolved for many years. After returning to Madras from Oxford, when Vijayaraghavan met Pillai at Madras, he quietly passed on Hardy's problem to Pillai. Pillai in his paper titled 'On the number of numbers which contain a fixed number of prime factors' (MS, 14, 1929, pp. 250-251) announced an important breakthrough to Hardy's problem. In 1943, as a faculty member of the University of Calcutta, Pillai started supervising a brilliant young student named L G Sathe. Sathe had obtained a research scholarship at Calcutta University. Pillai suggested the unsolved 'Hardy's Problem' to L G Sathe. He also gave him all the earlier manuscripts related to the problem. Noted number theorist, Professor R Balasubramanian has nicely described Sathe's contributions. He wrote:

\footnotetext{
“......In the course of less than two years, L G Sathe produced a monumentally complex induction argument, that ran into 134 printed pages when published, and that did much more than solve Hardy's problem. In particular, and for the first time, Sathe was able to show that Landau's asymptotic remained valid for $k<e \log \log x$. When finally in 1954, Sathe's result would supercede a theorem of Erdôs, which appeared in 1948 and which solves Hardy's problem by obtaining Landau's asymptotic for $k$ in an interval of length about $(\log \log x)^{1 / 2}$ around $\log \log x$. The sequence of events leading to publication of Sathe's work however remains somewhat opaque. It is clear, though, that Sathe submitted his work to 'Transactions of American Mathematical Society'..........that Selberg's comments on this work was sought by the editors of the Transactions. Finally, however, Sathe's work appeared in the 'Journal of the Indian Mathematical Society',........in four parts, the first part in 1953 and the last in 1954."
}

Unfortunately, Sathe fell seriously ill soon after and became incapacitated. So that cut short a 
brilliant mathematical talent, who might have contributed much more to the school of research of number theory in Bengal. Dr. Pillai also died of a plane crash in 1950 at the age of 49 . These two major disasters practically stalled any further progress in research on theory of numbers in Bengal.

\section{Trends of Research in Bihar}

In Bihar, the first paper on number theory was published in the late forties of the twentieth century. It was authored by S D Upadhyay and P N Dasgupta, both were attached to the Patna Science College. The paper titled 'On a generalized continued fraction' (BCMS, 39(1-4), 1947, pp. 65-70) deals with continued fractions. In this paper the authors state that a series of expressions for the numerator and denominator of any convergent of a continued fraction by means of matrices had been given by Milne-Thomson in 1933. The authors of this paper while working on a problem related to reciprocal differences in connection with interpolation formula had to consider continued fractions of a general type where coefficients themselves represent continued fractions. A year later guided by P N Dasgupta, Anunoy Chatterjee also from the Science College of the Patna University published a paper titled 'On a continued fraction of a general type' (BCMS, 40,1948 , pp. 69-75). It is stated by the author in this paper that Milne-Thomson in 1933 first exhibited a simple continued fraction in the form of a continued matrix product. Upadhyay and Dasgupta in their 1947 (the paper already mentioned above) discussed continued fractions of a general type where various partial quotients were themselves continued fractions. In this paper the author A Chatterjee has discussed some of the formulae obtained by Upadhyay and Dasgupta and has extended the idea of continuing the type referred to by further assuming the partial quotients of the general type as being expressible themselves by continued fractions.
From the late sixties of the twentieth century, a number of mathematicians in Bihar contributed research papers on various topics of number theory. T N Sinha published a paper titled 'Some systems of Diophantine equations of the TerryEscott type' (JIMS, NS, 30.1, 1966, pp. 15-26). M R Iyer in 1969 published a series of three papers related to Fibonacci numbers and they were all published in FQJ. In $1971 \mathrm{~T}$ N Sinha again published a paper on integer solutions of equations of a special type and it was published in MS. The other researchers from Bihar who published during the latter half of the twentieth century include S A N Moorthy, P D Shukla, R Tandon, S N Prasad, R N Lal, A Ahmad, K C Prasad, R N Singh, D N Singh, S N Dubey and A Murthy. These mathematicians have worked on topics ranging from prime numbers, Diophantine equations, quadratic forms to divisibility of numbers.

\section{ACKNOWLEDGement}

The author expresses her sincere gratitude to the Indian National Science Academy (INSA) for providing financial support for the project entitled "The Development of the School of Research on Number Theory in India During the $20^{\text {th }}$ Century". The present article is based on the project related work. The author conveys her thanks to Dr. Pradip Kumar Majumder of the Rabindra Bharati University for providing important references.

\section{Appendices}

1. Waring's Problem: British mathematician and Lucasian Professor of Mathematics in the University of Cambridge, Edward Waring (1736-1798) conjectured in 1770 that every natural number natural number can be represented as the sum of 4 squares, 9 cubes, 19 biquadrates and 'so on'. It was interpreted that the latter means, for every integer $k \geq 2$ there exists an integer $s=s(k)$ such that every natural number $\mathrm{n}$ can be expressed in the form $x_{1}^{k}+x_{2}^{k}+\ldots \ldots+x_{s(k)}^{5}$ with $x_{1}, x_{2}, \ldots \ldots x_{s}$, non-negative integers. It is conventional to denote least such $s$ by $\mathrm{g}(\mathrm{k})$. Thus $\mathrm{g}(2)=4$. Waring's conjecture was proved by Hilbert in 1909. Another quite 
different proof was given by Hardy and Littlewood in 1920.

2. Ramanujan's Function $\tau(\mathrm{n})$ : Definition of tau $(\mathrm{n})$ is as follows:

This is an integer valued function on natural numbers.

Mathematically speaking, a function $\tau$ (n) is related to the divisor function $\sigma_{\mathrm{r}}(\mathrm{n})$, where a divisor function is an arithmetic function giving the number of divisors of an integer. Tau function is sometimes also called Ramanujan's tau function. This function gives Fourier coefficients of Ramanujan modular form. Symbolically it is written as

$$
\begin{aligned}
g(x) & =x\left\{(1-x)\left(1-x^{2}\right)\left(1-x^{3}\right) \ldots \ldots . .\right\}^{24} \\
& =\sigma_{r}(n)=x \prod_{n=1}^{\infty}\left(1-x^{n}\right)^{24}=\sum_{n=1}^{\infty} \tau(n) x^{n}
\end{aligned}
$$

where $\mathrm{g}(\mathrm{x})$ is the generating function of the $\tau$-function.

3. Diophantine Equation: Any equation in one or more unknowns having solutions in integers is called a Diophantine Equation. It is named after the famous Greek mathematician Diophantus.

4. Theory of Partitions: A partition of an integer $n$ is a division of $\mathrm{n}$ into any number of positive integral parts. The number of partitions of $n$ is denoted by $p(n)$. For example,

$p(1)=1$ and $1=1, p(2)=2$ and $2=2,1+1, p(3)=3$ and $3=3,2+1,1+1+1, p(4)=5$ and $4=4,3+1,2$
$+2,2+1+1,1+1+1+1$, etc. The theory dealing with such functions is called the 'Theory of Partitions'.

\section{Abbreviations}

AMM: American Mathematical Monthly

BCMS: Bulletin of the Calcutta Mathematical Society

FQJ: Fibonacci Quarterly Journal

JIMS (NS): Journal of the Indian Mathematical Society (New Series)

JLMS: Journal of the London Mathematical Society

PBMS: Proceedings of the Benares Mathematical Society

PJM: Pacific Journal of Mathematics

PNAS (India): Proceedings of the National Academy of Sciences (India)

QJM: Quarterly Journal of Mathematics.

\section{BIBLIOGRAPHY}

Choudhury, A. 'Deba Brata Lahiri', Biographical Memoirs, Indian National Science Academy, 2003. 